\title{
ON ACYCLIC SIMPLICIAL COMPLEXES
}

\author{
FRANK HARARY AND EDGAR M. PALMER
}

The higher dimensional concepts corresponding to trees are developed and studied. In order to enumerate these 2-dimensional structures called 2-trees, a dissimilarity characteristic theory is investigated. By an appropriate application of certain combinatorial techniques, generating functions are obtained for the number of 2-trees. These are specialized to count those 2-trees embeddable in the plane, thus providing a new approach to the old problem of determining the number of triangulations of a polygon.

1. Pure Complexes. By an $n$-complex, we mean a finite $n$-dimensional simplicial complex. One of us $[3$, p. 462] defined a pure $n$-complex as an $n$-complex in which every $k$-simplex with $k<n$ is contained in an $n$-simplex. A pure $n$-complex will be called an $n$-plex for brevity.

We will only be concerned with 2-plexes, and for convenience 0-simplexes, 1 -simplexes and 2-simplexes are called points, lines and cells respectively. We now define for 2-plexes the analogues of the concepts of walk, trail, and path in graph theory [5]. In a given 2-plex $P$ a 1, 2-walk is an alternating sequence $x_{0}, \sigma_{1}$, $x_{1}, \sigma_{2}, x_{2}, \ldots, x_{n-1}, \sigma_{n}, x_{n}$ of lines $x_{i}$ and cells $\sigma_{j}$ such that each line $x_{i}$ is incident with the cells $\sigma_{i}$ and $\sigma_{i+1}$. This walk is closed if $x_{0}=x_{n}$, and is open otherwise. If all of the cells are distinct, it is called a 1,2-trail; if both the lines and the cells are distinct, it is a 1, 2-path.

A 2-plex is 1, 2-connected if every pair of lines are joined by a 1, 2-path. It is called 1, 2-acyclic if it contains no closed 1, 2-trails with more than one cell. For brevity we will say that a 2-plex is connected if it is 1, 2-connected; acyclic if 1, 2-acyclic. Note that if a 2-plex is connected, so is its underlying graph (in the usual sense), but not conversely. A 2-plex is simply connected if it is connected and its first Betti number is zero; intuitively, it has no "holes".

2. 2-trees. In the study of 2-plexes, the 2-tree plays the role of the tree in graph theory. In this section we present some of the important properties of 2-trees. Observe that each of these corresponds to a similar property of trees.

A 2-tree is a simply connected, acyclic 2-plex. The eccentricity of a line $x$ in a 2-tree is the number of cells in a longest 1,2-path beginning with $x$. The centre of a 2-tree is the subplex induced by all lines of minimum eccentricity. An end-cell has two lines incident with no other cells. The next three propositions follow readily from these definitions.

Proposition 1. The centre of a 2-tree is a line or a cell.

The proof is analogous to that of the corresponding proposition for trees: the centre of any tree is a point or a line; see König [8, p. 65].

Research supported by grants from the U.S. Air Force Office of Scientific Research and the U.S. Office of Naval Research.

[MATHEMATIKA 15 (1968), 115-122] 
Proposition 2. Every 2-tree with at least two cells has at least two end-cells.

It is well known that every nontrivial tree (with more than one point) has at least two endpoints. The proof depends on the finiteness of a tree and its acyclicity, as does the proof of this proposition.

Let $p, q$, and $r$ be the number of points, lines, and cells of a 2-plex.

Proposition 3. For any 2-tree, the following equations hold:

$$
\begin{aligned}
p-q+r & =1, \\
q-2 r & =1, \\
p-r & =2, \\
2 p-q & =3
\end{aligned}
$$

Obviously any two of these four equations imply the other two. The first of these is, of course, a variant of the Euler-Poincaré equation, and reduces to the familiar $p-q=1$ for trees. The second is easily verified by induction.

As in the case of trees, there are many alternate definitions for 2-trees. Two characterizations are given in the next proposition.

Proposition 4. The following are equivalent for a 2-plex $T$ :

(i) $T$ is a 2-tree;

(ii) $p-q+r=1$ and $T$ is connected and acyclic;

(iii) $p-r=2$ and $T$ is connected and acyclic.

That (i) implies (ii) and (iii) follows from Proposition 3 and the definition of 2-tree. To demonstrate that (ii) or (iii) implies (i), we show that $T$ is simply connected. This follows at once from the fact that for any 2-plex which is not simply connected, $p-r>2$ and $p-q+r<1$.

3. Dissimilarity characteristic theorem. Let $\alpha$ be a 1-1 map which sends the points, lines, and cells of one 2-plex onto the points, lines, and cells respectively of another 2-plex. If $\alpha$ preserves the incidence relations, then $\alpha$ is called an isomorphism. As usual, an automorphism is an isomorphism of a 2-plex with itself. The automorphisms of a 2-plex $P$ form a permutation group denoted $\Gamma(P)$ called the group of $P$. We shall refer to the orbits of this group as similarity classes. Thus two points (lines, cells) are similar whenever they are in the same similarity class, i.e. there is an automorphism which sends one point (line, cell) onto the other.

A homomorphism of the group of a 2-tree is obtained by restricting the automorphisms to the points of the centre. The image of this homomorphism is called the group of the centre of the 2-tree, and according as the centre is a line or a cell (Proposition 1), it is a subgroup of one of the symmetric groups $S_{2}$ or $S_{3}$.

Corresponding to the three partitions of the number 3 , there are three types of cells in a 2-tree:

Type (1), exactly two of its lines are similar;

Type (2), all three lines are similar;

Type (3), no two lines are similar. 
For a given 2-tree, we let $s_{1}$ and $s_{2}$ be the number of similarity classes of type (1) and type (2) respectively. Then we let

$$
s=s_{1}+2 s_{2} \text {. }
$$

The following three observations about the three types of cells are obvious but important.

Proposition 5. If a 2-tree $T$ contains a cell uvw of type (1) in which $u v$ and $v w$ are similar, then line $u w$ is in the centre of $T$.

Proposition 6. If a 2-tree $T$ contains a cell of type (2), then that cell is the centre of $T$ and $s_{2}=1$.

Proposition 7. If the group of the centre of a 2-tree is the identity, then every cell is of type (3) and $s=0$.

By the number of dissimilar points $p^{*}$ of a 2-tree we mean the number of similarity classes of points; analogous definitions are made for the number $q^{*}$ of dissimilar lines and $r^{*}$ for cells. Otter's dissimilarity characteristic theorem for trees [9] provides the key to the enumeration of trees in terms of rooted trees. The next theorem performs the same function for 2-trees. Other dissimilarity characteristic results have been studied by Harary and Norman [6].

Proposition 8. (Dissimilarity Characteristic Theorem.) For any 2-tree with $q^{*}$ dissimilar lines, $r^{*}$ dissimilar cells, $s_{1}$ cells with two similar lines, $s_{2}$ cells with all three lines similar, and $s=s_{1}+2 s_{2}$,

$$
q^{*}+s-2 r^{*}=1 \text {. }
$$

Otter's proof of his dissimilarity characteristic theorem for trees was based on the fact that for any tree, the identification of its similar lines results in a tree whose number of points and lines leads to his equation. Similarly, given any 2-tree $T$, one can show that a 2-tree $T^{*}$ is obtained by identifying similar cells of $T$. Then one can readily see that $T^{*}$ has $q^{*}+s$ lines and $r^{*}$ cells. On applying Proposition 3 to the 2 -tree $T^{*}$, the resulting equation is (6).

4. Enumeration of 2-trees. In order to describe the counting of 2-trees, we require the usual notation of combinatorial enumeration theory, as given in Pólya [10], in [3, 4, 7] and especially in [5]. This includes the symbols $Z(A)$ for the cycle index of the permutation group $A$ and $Z(A, f(x))$ for the power series obtained when a series $f(x)$ is substituted into $Z(A)$. A familiarity with Pólya's classical enumeration theorem must be assumed. Three particular formulas which occur in 2-tree enumeration involve the substitution of series into the cycle indexes of the symmetric groups $S_{2}$ and $S_{3}$, and of the alternating group $A_{2}$ (actually the identity group of degree 2) in the form $Z\left(A_{2}-S_{2}\right)=Z\left(A_{2}\right)-Z\left(S_{2}\right)$. To be explicit, we now list the results of such substitutions:

$$
\begin{gathered}
Z\left(S_{2}, f(x)\right)=\frac{1}{2}\left[f^{2}(x)+f\left(x^{2}\right)\right], \\
Z\left(S_{3}, f(x)\right)=\frac{1}{6}\left[f^{3}(x)+3 f(x) f\left(x^{2}\right)+2 f\left(x^{3}\right)\right], \\
Z\left(A_{2}-S_{2}, f(x)\right)=\frac{1}{2}\left[f^{2}(x)-f\left(x^{2}\right)\right] .
\end{gathered}
$$


In equation (9), $Z\left(A_{2}-S_{2}\right)$ appears as a special case of a result of Pólya [10] to the effect that $Z\left(A_{n}-S_{n}\right)$ enumerates those configurations in which the figures are all distinct. Finally we state the useful identity:

$$
\sum_{n=0}^{\infty} Z\left(S_{n}, f(x)\right)=\exp \sum_{r=1}^{\infty} \frac{f\left(x^{r}\right)}{r},
$$

which sums the cycle indexes of all the symmetric groups.

Now we proceed to develop the generating functions for 2-trees. Let $t_{n}$ be the number of 2-trees with $n$ cells. The counting series for 2-trees is denoted by

$$
t(x)=\sum_{n=1}^{\infty} t_{n} x^{n} .
$$

In order to derive formulas for $t_{n}$ we will make use of the corresponding series for various kinds of rooted 2-trees.

First let $M_{1}(x)$ and $N_{1}(x)$ be the series for 2-trees rooted at a symmetric and an unsymmetric end-line respectively. Further, let $M(x)$ and $N(x)$ be the series for 2 -trees rooted at any symmetric and any unsymmetric line respectively. The following two equations express $M_{1}(x)$ and $N_{1}(x)$ in terms of $M(x)$ and $N(x)$ :

$$
\begin{gathered}
M_{1}(x)=x\left(1+M\left(x^{2}\right)+2 N\left(x^{2}\right)\right), \\
N_{1}(x)=x Z\left(A_{2}-S_{2}, 1+M(x)+2 N(x)\right) .
\end{gathered}
$$

Equation (13) can be stated more explicitly by applying (9). Next we express $M(x)$ in terms of $M_{1}(x)$ and $N_{1}(x)$ :

$$
M(x)=\sum_{n=1}^{\infty} Z\left(S_{n}, M_{1}(x)+N_{1}\left(x^{2}\right)\right) .
$$

Using the identity (10), equation (14) may be written:

$$
M(x)=-1+\exp \left\{\sum_{n=1}^{\infty} \frac{1}{n}\left[M_{1}\left(x^{n}\right)+N_{1}\left(x^{2 n}\right)\right]\right\} .
$$

Now note that the counting series for 2-trees rooted at an oriented line is simply $M(x)+2 N(x)$. From this observation we have:

$$
M(x)+2 N(x)=\sum_{n=1}^{\infty} Z\left(S_{n}, M_{1}(x)+2 N_{1}(x)\right) .
$$

Again using the identity (10) we may write (16) as

$$
M(x)+2 N(x)=-1+\exp \left\{\sum_{n=1}^{\infty} \frac{1}{n}\left[M_{1}\left(x^{n}\right)+2 N_{1}\left(x^{n}\right)\right]\right\} .
$$

Thus equations (15) and (17) may be used to solve for $N(x)$ in terms of $M_{1}(x)$ and $N_{1}(x)$. Now using all four formulas (12), (13), (15) and (17), the coefficients of $M(x)$ and $N(x)$ can be calculated. For the first few terms we have:

$$
\begin{gathered}
M(x)=x+x^{2}+2 x^{3}+3 x^{4}+6 x^{5}+\ldots \\
N(x)=x^{2}+4 x^{3}+18 x^{4}+77 x^{5}+\ldots
\end{gathered}
$$


The series for 2-trees rooted at a line is denoted $L(x)$ and since $L(x)=M(x)+N(x)$, we have immediately:

$$
L(x)=x+2 x^{2}+6 x^{3}+21 x^{4}+83 x^{5}+\ldots .
$$

We denote the series for 2 -trees rooted at a cell (triangle) by $\Delta(x)$. It can be shown that

$$
\Delta(x)=x Z\left(S_{3}, 1+M(x)+2 N(x)\right)-x N(x)\left(1+M\left(x^{2}\right)+2 N\left(x^{2}\right)\right) .
$$

Having expressed $\Delta(x)$ in terms of $M(x)$ and $N(x)$, we substitute (18) and (19) in equation (21) to obtain

$$
\Delta(x)=x+x^{2}+3 x^{3}+10 x^{4}+39 x^{5}+\ldots
$$

Following the notation of the Dissimilarity Characteristic Theorem, we denote by $s_{1}(x)$ the counting series for 2-trees rooted at a cell of type (1). Similarly $s_{2}(x)$ is the series for 2-trees rooted at a cell of type (2). These two series are readily expressed as functions of $M_{1}(x), M(x)$ and $N(x)$ :

$$
\begin{gathered}
s_{1}(x)=M_{1}(x)(1+M(x))-x\left(1+M\left(x^{3}\right)\right), \\
s_{2}(x)=x\left(1+M\left(x^{3}\right)+N\left(x^{3}\right)\right) .
\end{gathered}
$$

Making the appropriate substitutions we obtain

$$
\begin{gathered}
s_{1}(x)=x^{2}+2 x^{3}+2 x^{4}+7 x^{5}+\ldots, \\
s_{2}(x)=x+x^{4}+2 x^{7}+6 x^{10}+\ldots .
\end{gathered}
$$

In order to obtain the formula for $t(x)$, the series for 2-trees, we use the Dissimilarity Characteristic Theorem in the same manner as was done by Otter [9] for the enumeration of trees. If we sum equation (6) over all 2-trees with a given number $n$ of cells, we find that the total number of such trees is

$$
t_{n}=\Sigma q^{*}+\Sigma s-2 \Sigma r^{*}
$$

But then $\Sigma q^{*}$ is just the coefficient of $x^{n}$ in $L(x)$, while $\Sigma s$ is the coefficient of $x^{n}$ in $s_{1}(x)+2 s_{2}(x)$, and $\Sigma r^{*}$ is the coefficient of $x^{n}$ in $\Delta(x)$. This result is summarized by the following theorem.

PROPOSITION 9. (Enumeration Theorem for 2-trees.) The counting series for 2-trees is

$$
t(x)=L(x)+s_{1}(x)+2 s_{2}(x)-2 \triangle(x) .
$$

Substituting equations $(20,22,25,26)$ into equation $(28)$ gives

$$
t(x)=x+x^{2}+2 x^{3}+5 x^{4}+12 x^{5}+\ldots
$$

Note that this theorem can be used to count 2-trees with specified properties provided that formulas for $L(x), s_{1}(x), s_{2}(x)$ and $\Delta(x)$ are found for 2-trees with these properties. The corresponding investigation for trees of various species was made by Harary and Prins [7].

5. On the number of triangulations of a polygon. By a triangulation of a polygon we mean a graph obtained from a regular $n$-gon by adding non-intersecting chords until every interior region is a triangle. Obviously $n-3$ chords are required and $n-2$ triangles are obtained. Generating functions for the number of different triangula- 
tions of the $n$-gon, i.e. those not isomorphic as graphs, have been found by Brown [1], but our purpose here is to present an entirely different approach toward finding such a generating function. We alter the formulation of the problem into a statement involving 2-dimensional simplicial complexes by observing that triangulations of a polygon correspond precisely with planar 2-trees. We then proceed to enumerate the latter by the same methods exploited for counting 2-trees.

To illustrate the configurations being counted, we show in Figure 1 the unique triangulations of a triangle, a quadrilateral, and a pentagon, and the three different triangulations of a hexagon. Note that these graphs are not taken as rooted or labelled in any way. Observe also the correspondence between these and the planar 2-trees with one, two, three and four cells.
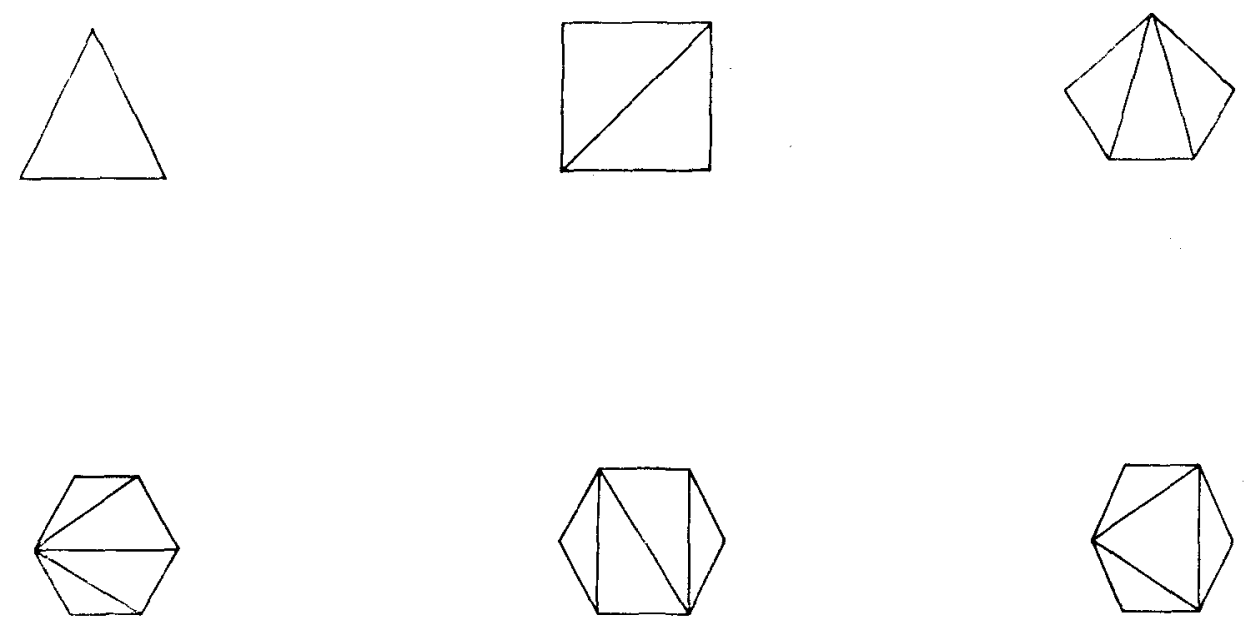

FIG. 1.-The triangulations of the $n$-gon, $n=3$ to 6 .

The enumeration of planar 2-trees can be accomplished by using almost all of the formulas that have already been developed for 2-trees. Therefore we alter the notation used for 2-trees only slightly by writing a bar to indicate the generating functions for planar 2-trees.

Thus let $\bar{M}_{1}(x)$ and $\bar{N}_{1}(x)$ be the series for planar 2-trees rooted at a symmetric and an unsymmetric end-line respectively. Then the following two formulas (compare (12) and (13)) specify the relationship between $\bar{M}_{1}(x)$ and $\bar{N}_{1}(x)$.

$$
\begin{gathered}
\bar{M}_{1}(x)=x\left(1+\bar{M}_{1}\left(x^{2}\right)+2 \bar{N}_{1}\left(x^{2}\right)\right), \\
\bar{N}_{1}(x)=x Z\left(A_{2}-S_{2}, 1+\bar{M}_{1}(x)+2 \bar{N}_{1}(x)\right) .
\end{gathered}
$$

These two equations (30) and (31) can be used to obtain the coefficients in the two series $\bar{M}_{1}(x)$ and $\bar{N}_{1}(x)$ by exhaustion, as for (12) and (13). However, as Brown [2, p. 752] pointed out, a formula due to Euler (and repeatedly rediscovered) shows that the number of triangulations of an $(n+2)$-gon which is rooted by orienting one of its boundary edges (and hence the polygon) is

$$
f_{n}=\frac{2(2 n-1) !}{(n-1) !(n+1) !}=\frac{1}{n}\left(\begin{array}{c}
2 n \\
n-1
\end{array}\right) \text {. }
$$


Hence it follows that $\bar{M}_{1}(x)+2 \bar{N}_{1}(x)=\Sigma f_{n} x^{n}$, so that

$$
\bar{M}_{1}(x)+2 \bar{N}_{1}(x)=\sum_{n=1}^{\infty} \frac{2(2 n-1) !}{(n-1) !(n+1) !} x^{n}
$$

Now from Euler's formula (32) and equation (30) for $\bar{M}_{1}(x)$ we have

$$
\bar{M}_{1}(x)=x+\sum_{n=1}^{\infty} \frac{2(2 n-1) !}{(n-1) !(n+1) !} x^{2 n+1} .
$$

For the first few terms of $\bar{M}_{1}(x)$ and $\bar{N}_{1}(x)$ we have:

$$
\begin{gathered}
\bar{M}_{1}(x)=x+x^{3}+2 x^{5}+5 x^{7}+14 x^{9}+\ldots \\
\bar{N}_{1}(x)=x^{2}+2 x^{3}+7 x^{4}+20 x^{5}+66 x^{6}+212 x^{7}+715 x^{8}+\ldots
\end{gathered}
$$

The series for planar 2-trees rooted at a line is denoted $L(x)$ and can be expressed in terms of $\bar{M}_{1}(x)$ and $\bar{N}_{1}(x)$ :

$$
\bar{L}(x)=Z\left(S_{2}, 1+\bar{M}_{1}(x)+\bar{N}_{1}(x)\right)+Z\left(S_{2}, \bar{N}_{1}(x)\right)-1 .
$$

Substitution of (35) and (36) in equation (37) for $\bar{L}(x)$ yields

$$
L(x)=x+2 x^{2}+4 x^{3}+12 x^{4}+34 x^{5}+111 x^{6}+360 x^{7}+1226 x^{8}+\ldots
$$

From this point on, since the equations and procedure are virtually the same for planar 2-trees as for 2-trees, we will simply list the formulas for $\bar{\triangle}(x), \bar{s}_{1}(x), \bar{s}_{2}(x)$, and $\bar{t}(x)$. We have

$$
\begin{gathered}
\bar{\triangle}(x)=x Z\left(S_{3}, 1+\bar{M}_{1}(x)+2 \bar{N}_{1}(x)\right)-x \bar{N}_{1}(x)\left(1+\bar{M}_{1}\left(x^{2}\right)+2 \bar{N}_{1}\left(x^{2}\right)\right) \\
\bar{s}_{1}(x)=\bar{M}_{1}(x)\left(1+\bar{M}_{1}(x)\right)-x\left(1+\bar{M}_{1}\left(x^{3}\right)\right) \\
\bar{s}_{2}(x)=x \bar{N}_{1}\left(x^{3}\right)+x\left(1+\bar{M}_{1}\left(x^{3}\right)\right)
\end{gathered}
$$

and as before

$$
\bar{t}(x)=\bar{L}(x)+\bar{s}_{1}(x)+2 \bar{s}_{2}(x)-2 \bar{\Delta}(x)
$$

which is obtained by barring equation (28), the Enumeration Theorem for 2-trees.

Substituting the calculations (35) and (36) for $\bar{M}_{1}(x)$ and $\bar{N}_{1}(x)$ in these formulas gives

$$
\bar{t}(x)=x+x^{2}+x^{3}+3 x^{4}+4 x^{5}+12 x^{6}+27 x^{7}+82 x^{8}+\ldots
$$

for the number of planar 2-trees.

In an unpublished manuscript, R. K. Guy has worked further with the results of Brown [1] and (with the help of a computing machine) obtains several additional coefficients for $\bar{t}(x)$ :

$$
\begin{aligned}
\bar{t}(x)= & x+x^{2}+x^{3}+3 x^{4}+4 x^{5}+12 x^{6}+27 x^{7}+82 x^{8}+228 x^{9}+733 x^{10} \\
& +2282 x^{11}+7528 x^{12}+24,834 x^{13}+83,898 x^{14}+285,357 x^{15} \\
& +1,046,609 x^{16}+3,412,420 x^{17}+11,944,614 x^{18}+42,080,170 x^{19} \\
& +149,197,152 x^{20}+531,883,768 x^{21}+1,905,930,975 x^{22} \\
& +6,861,221,666 x^{23}+\ldots
\end{aligned}
$$




\section{References}

1. W. G. Brown, " Enumeration of triangulations of the disk", Proc. London Math Soc., 14 (1964), 746-768.

2. _____ " "Historical note on a recurrent combinatorial problem", Amer. Math. Monthly, 72 (1965), 973-977.

3. F. Harary, "The number of linear, directed, rooted, and connected graphs", Trans. Amer. Math. Soc., 78 (1955), 445-463.

4. _ـ "Combinatorial problems in graphical enumeration ", Chapter 6 in Applied Combinatorial Mathematics, E. F. Beckenbach, ed. (New York, Wiley, 1964), 185-217.

5. - - A seminar on graph theory (New York, Holt, Rinehart and Winston, 1967), 1-41.

6. - and R. Z. Norman, "Dissimilarity characteristic theorems for graphs", Proc. Amer. Math. Soc., 11 (1960), 332-334.

7. _ and G. Prins, "The number of homeomorphically irreducible trees and other species ", Acta Math., 101 (1959), 141-162.

8. D. König, Theorie der endlichen und unendlichen Graphen (Leipzig, 1936; reprinted New York, Chelsea, 1950).

9. R. Otter, “The number of trees ", Ann. of Math., 49 (1948), 583-599.

10. G. Pólya, "Kombinatorische Anzahlbestimmungen für Gruppen, Graphen und chemische Verbindungen ", Acta Math., 68 (1937), 145-254.

University of Michigan,

University College, London. 